\title{
UNIQUENESS AND ORDER IN SEQUENTIAL EFFECT ALGEBRAS
}

\author{
Stan Gudder \\ Department of Mathematics \\ University of Denver \\ Denver, Colorado 80208 \\ sgudder@math.du.edu
}

\author{
Richard Greechie \\ Department of Mathematics \\ Louisiana Tech University \\ Ruston, Louisiana 71272 \\ greechie@math.latech.edu
}

\begin{abstract}
A sequential effect algebra (SEA) is an effect algebra on which a sequential product is defined. We present examples of effect algebras that admit a unique, many and no sequential product. Some general theorems concerning unique sequential products are proved. We discuss sequentially ordered SEA's in which the order is completely determined by the sequential product. It is demonstrated that intervals in a sequential ordered SEA admit a sequential product.
\end{abstract}

\section{Introduction}

Quantum effects are a basic concept in foundational studies of quantum physics [2, 3, 4, 过, 6]. Quantum effects correspond to yes-no measurements that may be unsharp and in recent years they have been studied within a general algebraic framework called an effect algebra [1, 6, 7, 8, 14]. Although effect algebras have been useful for our understanding of quantum theory they appear to be too general. Effect algebras only describe one measurement connective OR (denoted by $\oplus$ ) and a negation NOT (denoted by ' ${ }^{\prime}$ ). Roughly speaking, $\oplus$ represents a parallel measurement of two effects. However, it is important to have a mechanism for describing series or sequential measurements of effects (denoted by $\circ$ ). For this reason the authors have introduced a structure called a sequential effect algebra (SEA) 10, 13. A SEA is an effect algebra on which a sequential product $\mathrm{o}$ with natural properties is defined. These properties hold in the important case of Hilbert space effect algebras [11, 12].

The present paper concentrates on uniqueness and order properties of SEA's. We shall show that some effect algebras admit a unique sequential product, others admit many and still others admit none. We also present some general results on the uniqueness of sequential products. We then discuss a class of SEA's in which the order is completely determined by the sequential product. We call 
this class sequentially ordered SEA's. We give examples of SEA's that are sequentially ordered and examples that are not. We finally show that intervals in a sequentially ordered SEA admit a sequential product. Although we review some of the basic properties of effect algebras and SEA's we refer the reader to our cited literature for more details.

\section{Effect Algebras}

An effect algebra is an algebraic system $(E, 0,1, \oplus)$ where $0 \neq 1 \in E$ and $\oplus$ is a partial binary operation on $E$ satisfying:

(A1) If $a \oplus b$ is defined, then $b \oplus a$ is defined and $b \oplus a=a \oplus b$.

(A2) If $a \oplus b$ and $(a \oplus b) \oplus c$ are defined, then $b \oplus c$ and $a \oplus(b \oplus c)$ are defined and $a \oplus(b \oplus c)=(a \oplus b) \oplus c$.

(A3) For every $a \in E$ there exists a unique $a^{\prime} \in E$ such that $a \oplus a^{\prime}=1$.

(A4) If $a \oplus 1$ is defined, then $a=0$.

If $a \oplus b$ is defined, we write $a \perp b$. We define $a \leq b$ if there exists a $c \in E$ such that $a \oplus c=b$. It can be shown that $\left(E, \leq,^{\prime}\right)$ is a poset with $0 \leq a \leq 1$ for every $a \in E, a^{\prime \prime}=a$, and $a \leq b$ implies $b^{\prime} \leq a^{\prime}$ [6, 7]. Also, $a \perp b$ if and only if $a \leq b^{\prime}$. If $a \oplus a \oplus \cdots \oplus a$ ( $n$ summands) is defined we denote this element by $n a$. An element $a \in E$ is sharp if $a \wedge a^{\prime}=0$. If every element of $E$ is sharp, then $E$ is an orthoalgebra. It is easy to show that $E$ is an orthoalgebra if and only if $a \perp a$ implies $a=0$.

Example 1. For a Boolean algebra $\mathcal{B}$, define $a \perp b$ if $a \wedge b=0$ and in this case $a \oplus b=a \vee b$. Then $(\mathcal{B}, 0,1, \oplus)$ is an effect algebra that happens to be an orthoalgebra. In particular, if $X \neq \emptyset$, then $\left(2^{X}, \emptyset, X, \oplus\right)$ is an effect algebra.

Example 2. For $[0,1] \subseteq \mathbb{R}$, define $a \perp b$ if $a+b \leq 1$ and in this case $a \oplus b=a+b$. Then $([0,1], 0,1, \oplus)$ is an effect algebra. The only sharp elements are 0 and 1 .

Example 3. Let $X \neq \emptyset$ and let $\mathcal{F} \subseteq[0,1]^{X}$. We call $\mathcal{F}$ a fuzzy set system on $X$ (i) if the functions $0,1 \in \mathcal{F}$, (ii) if $f \in \mathcal{F}$ then $1-f \in \mathcal{F}$, (iii) if $f, g \in \mathcal{F}$ with $f+g \leq 1$ then $f+g \in \mathcal{F}$ and (iv) if $f, g \in \mathcal{F}$ then $f g \in \mathcal{F}$. Then $(\mathcal{F}, 0,1, \oplus)$ is an effect algebra with $f \oplus g=f+g$ whenever $f+g \leq 1$. If $\mathcal{F}=[0,1]^{X}$ we call $\mathcal{F}$ a full fuzzy set system. The sharp elements of a fuzzy set system $\mathcal{F}$ are the characteristic functions in $\mathcal{F}$ which can be identified with the (sharp) subsets of $X$ in $\mathcal{F}$. Indeed, if $f \in \mathcal{F}$ is sharp, then $f(1-f) \leq f$, $1-f$ implies that $f(1-f)=0$. Hence, if $f(x) \neq 0$, then $f(x)=1$.

Example 4. Let $H$ be a Hilbert space and let $\mathcal{E}(H)$ be the set of self-adjoint operators on $H$ satisfying $0 \leq A \leq I$. For $A, B \in \mathcal{E}(H)$ we define $A \perp B$ if 
$A+B \in \mathcal{E}(H)$ and in this case $A \oplus B=A+B$. Then $(\mathcal{E}(H), 0, I, \oplus)$ is an effect algebra. The elements of $\mathcal{E}(H)$ are called quantum effects and are important in quantum measurement theory [2, 33, 4, 15, 16]. The sharp elements of $\mathcal{E}(H)$ are the set of projection operators $\mathcal{P}(H)$ on $H$.

Example 5. There are many examples of finite nonboolean effect algebras. The simplest example is the 3 -chain $C_{3}=\{0, a, 1\}$ where $2 a=1$. Another example is the diamond $D=\{0, a, b, 1\}$ where $2 a=2 b=1$.

Example 6. Let $E=\omega+\omega^{*}$ be the set of elements

$$
E=\left\{0, a, 2 a, \ldots,(2 a)^{\prime}, a^{\prime}, 1\right\}
$$

By convention $0 a=0$. Define $\oplus$ on $E$ by

$$
(m a) \oplus(n a)=(m+n) a
$$

and when $n \leq m$

$$
(m a)^{\prime} \oplus(n a)=(n a) \oplus(m a)^{\prime}=((m-n) a)^{\prime}
$$

then $(E, 0,1, \oplus)$ is an effect algebra.

Let $\left(E_{i}, 0_{i}, 1_{i}, \oplus_{i}\right)$ be a collection of effect algebras. One way of constructing a new effect algebra is by taking the cartesian product $\Pi E_{i}$ and defining $\Pi a_{i} \perp \Pi b_{i}$ if $a_{i} \perp b_{i}$ for every $i$ in which case $\Pi a_{i} \oplus \Pi b_{i}=\Pi\left(a_{i} \oplus b_{i}\right)$. Then $\left(\Pi E_{i}, \Pi 0_{i}, \Pi 1_{i}, \oplus\right)$ is an effect algebra. Another way is the horizontal sum construction $E=H S\left(E_{i}, i \in I\right)$ defined as follows. Identify all $0_{i}$ with a single element 0 and all the $1_{i}$ with a single element 1 . Let $E_{i}^{\prime}=E_{i} \backslash\left\{0_{i}, 1_{i}\right\}$, form the disjoint union $\dot{\cup} E_{i}^{\prime}$ and let $E=\{0,1\} \dot{\cup} E_{i}^{\prime}$. For $a, b \in E_{i}$ for some $i \in I$, if $a \perp b$ define $a \oplus b=a \oplus_{i} b$ and no other orthosums are defined on $E$. Then $(E, 0,1, \oplus)$ is an effect algebra. For example $D=H S\left(C_{3}, C_{3}\right)$. Finally, if $E$ is an effect algebra and $b \in E$ with $b \neq 0$, then the interval $[0, b]=\{a \in E: 0 \leq a \leq b\}$ can be organized into an effect algebra as follows. If $c, d \in[0, b]$ and $c \oplus d \leq b$ we define $c \oplus_{b} d=c \oplus d$. Then $\left([0, b], 0, b, \oplus_{b}\right)$ is an effect algebra.

\section{Sequential Effect Algebras}

For a binary operation $\circ$, if $a \circ b=b \circ a$ we write $a \mid b$. A sequential effect algebra (SEA) is an algebraic system $(E, 0,1, \oplus, \circ)$ where $(E, 0,1, \oplus)$ is an effect algebra and $\circ: E \times E \rightarrow E$ is a binary operation satisfying:

(S1) The map $b \mapsto a \circ b$ is additive for every $a \in E$, i.e., if $b \perp c$ then $a \circ b \perp a \circ c$ and $a \circ(b \oplus c)=a \circ b \oplus a \circ c$.

(S2) $1 \circ a=a$ for every $a \in E$.

(S3) If $a \circ b=0$, then $a \mid b$. 
(S4) If $a \mid b$, then $a \mid b^{\prime}$ and $a \circ(b \circ c)=(a \circ b) \circ c$ for every $c \in E$.

(S5) If $c \mid a$ and $c \mid b$, then $c \mid a \circ b$ and, when $a \perp b, c \mid(a \oplus b)$.

We call an operation satisfying (S1)-(S5) a sequential product on $E$. If $a \mid b$ for every $a, b \in E$, then $E$ is a commutative SEA. Notice that if $\circ$ is a commutative binary operation on an effect algebra $E$, to test whether $\circ$ is a sequential product we need only verify $(\mathrm{S} 1),(\mathrm{S} 2)$ and $a \circ(b \circ c)=(a \circ b) \circ c$.

Given an effect algebra $E$, does $E$ admit a sequential product and if so is it unique? We shall show that anything goes. There exist effect algebras that do not admit a sequential product. There are effect algebras that admit a unique sequential product and effect algebras that admit many sequential products.

Example 1 (continued). A Boolean algebra is a SEA under the operation $a \circ b=a \wedge b$. It is shown in 13] that $\circ$ is unique.

Example 2 (continued). The unit interval $[0,1] \subseteq \mathbb{R}$ is a SEA under the operation $a \circ b=a b$. We shall show later that $\circ$ is unique.

Example 3 (continued). A fuzzy set system $\mathcal{F}$ is a SEA under the operation $f \circ g=f g$. We shall show later that if $\mathcal{F}$ is full, then $\circ$ is unique.

Example 4 (continued). The effect algebra $\mathcal{E}(H)$ is a SEA under the operation $A \circ B=A^{1 / 2} B A^{1 / 2}$ [11, 12]. This SEA is important for quantum measurement theory [2, 3] and is our first example of a noncommutative SEA. We do not know whether $\circ$ is unique. However, as we shall later show, $\circ$ is unique if it satisfies some additional conditions.

Example 5 (continued). The effect algebras $C_{3}$ and $D$ do not admit sequential products. For $C_{3}$ suppose we have a sequential product $\circ$. Then

$$
a=a \circ 1=a \circ\left(a \oplus a^{\prime}\right)=a \circ a \oplus a \circ a^{\prime}=2(a \circ a)
$$

But there is no such element $a \circ a$ in $C_{3}$ which is a contradiction. A similar demonstration holds for $D$. More generally, it is shown in 13 that no nonboolean finite effect algebra admits a sequential product.

Example 6 (continued). It is shown in [13] that $E=\omega+\omega^{*}$ admits a unique sequential product. For $x, y \in E$ this sequential product is defined by

$$
x \circ y= \begin{cases}0 & \text { if } x=m a, y=n a \\ {[.25 p c] x \wedge y} & \text { if } x=m a, y=(n a)^{\prime} \text { or } x=(m a)^{\prime}, y=n a \\ {[.25 p c]((m+n) a)^{\prime}} & \text { if } x=(m a)^{\prime}, y=(n a)^{\prime}\end{cases}
$$

Let $\mathcal{D}(H)$ be the set of density operators on $H$. Notice that there exist $W \in \mathcal{D}(H)$ such that, for $A \in \mathcal{E}(H), \operatorname{tr}(W A)=0$ implies $A=0$. We call such a $W$ faithful. Indeed, let $x_{i}$ be an orthonormal basis for $H$ and denote the one-dimensional projection onto the span of $x_{i}$ by $P_{i}$. Then $W=\sum \lambda_{i} P_{i}$ where $\lambda_{i}>0, \sum \lambda_{i}=1$ is faithful. 
Example 7. This is an example of an effect algebra that admits many sequential products. Let $E_{1}=\mathcal{E}(H), E_{2}=[0,1] \subseteq \mathbb{R}$ and $E=H S\left(E_{1}, E_{2}\right)$. Define $\circ: E \times E \rightarrow E$ as follows. If $A, B \in E_{1}$ then $A \circ B=A^{1 / 2} B A^{1 / 2}$; if $a, b \in E_{2}$ then $a \circ b=a b$; if $A \in E_{1}, a \in E_{2}$ then $A \circ a=a A$; and if $a \in E_{2}$, $A \in E_{1}$ then $a \circ A=a \operatorname{tr}(W A)$ where $W \in \mathcal{D}(H)$ is fixed and faithful. It is shown in [13] that $\circ$ is sequential product on $E$. Notice that different faithful $W \in \mathcal{D}(H)$ give different sequential products.

Let $a$ be a sharp element of an effect algebra $E$. Suppose we view $a$ in a larger context by enlarging $E$ to an effect algebra $F$. Since $a$ may not be sharp as a member of $F$ we say that sharpness is contextual in effect algebras. Physically we would not expect sharpness to be contextual and this is an unfortunate property for effect algebras. The next result shows that this unfortunate property holds for any effect algebra that contains a nontrivial sharp element. The result also shows that sharpness is noncontextual for SEA's. We denote the set of sharp elements of an effect algebra $E$ by $E_{S}$. As usual, an embedding for an effect algebra is a monomorphism [6, 7].

Theorem 3.1. (i) If $E$ is an effect algebra, then there exists an effect algebra $F$ and an effect algebra embedding $\phi: E \rightarrow F$ such that $F_{S}=\{0,1\}$. (ii) If $E$ and $F$ are $S E A$ 's and $\phi: E \rightarrow F$ is a SEA embedding, then $\phi(a) \in F_{S}$ if and only if $a \in E_{S}$.

Proof. (i) Let $G$ be a nontrivial abelian partially ordered group. Define

$$
E_{G}=(E \backslash\{0,1\}) \times G \cup\{(0, g),(1,-g): g \geq 0\}
$$

and for $(a, g),(b, h) \in E_{G}$ define $(a, g) \oplus(b, h)=(a \oplus b, g+h)$ provided that $a \perp b$ and $(a \oplus b, g+h) \in E_{G}$. Letting $\mathbf{0}=(0,0), \mathbf{1}=(1,0)$, we shall show that $\left(E_{B}, \mathbf{0}, \mathbf{1}, \oplus\right)$ is an effect algebra. It is clear that (A1) (commutativity) holds. Defining $(a, g)^{\prime}=\left(a^{\prime},-g\right)$ it is easy to check that (A3) holds. To verify (A4), suppose that $(a, g) \oplus \mathbf{1}$ is defined. It follows that $a=0$ and $(1, g) \in E_{G}$. Since $(0, g) \in E_{G}$, it follows that $g=0$. Hence, $(a, g)=\mathbf{0}$.

To verify (A2) (associativity), assume that $(a, g),(b, h),(c, k) \in E_{G}$ with $(b, h) \oplus(c, k) \in E_{G}$ and

$$
(a, g) \oplus[(b, h) \oplus(c, k)] \in E_{G}
$$

Then

$$
\begin{aligned}
(a, g) \oplus[(b, h) \oplus(c, k)] & =(a \oplus(b \oplus c), g+(h+k)) \\
& =((a \oplus b) \oplus c,(g+h)+k) \\
& =(a \oplus b, g+h) \oplus(c, k)
\end{aligned}
$$

provided that $(a \oplus b, g+h) \in E_{G}$. Noting that this holds for all $g, h \in G$ when $a \oplus b \notin\{0,1\}$, we need only consider the cases $a \oplus b \in\{0,1\}$. If $a \oplus b=0$, then $a=b=0$. Hence, $g, h \geq 0$ so that $(a \oplus b, g+h) \in E_{G}$. If $a \oplus b=1$, then $c=0$ 
so that $k \geq 0$. Also, by $(*)$, we have $(g+h)+k \leq 0$ so that $g+h \leq-k \leq 0$. Hence, $(a \oplus b, g+h) \in E_{G}$ so $\left(E_{G}, \mathbf{0}, \mathbf{1}, \oplus\right)$ is an effect algebra.

Note that $(a, g) \leq(b, h)$ in $E_{G}$ if and only if $a \leq b$ and $(b \ominus a, h-g) \in E_{G}$. We thus have either $a<b$ or $a=b$ and $g \leq h$ which is the lexicographic order on $E_{G}$. Define $\phi: E \rightarrow E_{G}$ by $\phi(a)=(a, 0)$. Clearly $\phi$ is an effect algebra embedding of $E$ into $E_{G}$. To show that $\left(E_{G}\right)_{S}=\{\mathbf{0}, \mathbf{1}\}$ suppose $(a, g) \in E_{G}$ with $a \neq 0,1$. Then for $h \in G$ with $h>0$ we have $\left.(0, h) \leq(a, g), a^{\prime},-g\right)$. Hence, $(a, g) \notin\left(E_{G}\right)_{S}$. For the case $(0, g) \in E_{G}$ with $g>0$ we have $(0, g)<(1,-g)$ and for the case $(1, g) \in E_{G}$ with $g<0$ we have $(0,-g)<(1, g)$.

(ii) If $a \in E_{S}$, then $\phi(a) \circ \phi(a)=\phi(a \circ a)=\phi(a)$ so that $\phi(a) \in F_{S}$. Conversely, if $a \notin E_{S}$ then there exists a $b \in E$ such that $0<b \leq a, a^{\prime}$. But then $0<\phi(b) \leq \phi(a), \phi(a)^{\prime}$ so that $\phi(a) \notin F_{S}$.

The second part of the proof of Theorem 3.1(ii) shows that fuzziness is noncontextual in an effect algebra $E$. That is, if $\phi: E \rightarrow F$ is an effect algebra embedding and $a \notin E_{S}$ then $\phi(a) \notin F_{S}$. The construction of the effect algebra $E_{G}$ in the proof of Theorem 3.1 (i) is of interest in its own right. The elements of the form $(0, g) \in E_{G}$ act like infinitesimals. In the special case where $E=\{0,1\}$ is trivial and $G$ is the integers $Z$, we have that $E_{Z}$ is isomorphic to $\omega+\omega^{*}$.

\section{Results}

Theorem 4.1. There is a unique sequential product on the effect algebra $[0,1] \subseteq$ $\mathbb{R}$.

Proof. Let $\circ$ be a sequential product on $[0,1]$. Then for any integer $n \geq 1$ and $a \in[0,1]$ we have

$$
a=a \circ 1=a \circ\left(\frac{1}{n} \oplus \cdots \oplus \frac{1}{n}\right)=n\left(a \circ \frac{1}{n}\right)
$$

so that $a \circ \frac{1}{n}=\frac{1}{n} a$. Also, for any integer $1 \leq m \leq n$ we have

$$
a \circ \frac{m}{n}=a \circ\left(\frac{1}{n} \oplus \cdots \oplus \frac{1}{n}\right)=m\left(a \circ \frac{1}{n}\right)=\frac{m}{n} a
$$

Hence, for any rational number $r \in Q \cap[0,1]$ we have $a \circ r=a r$. Now let $b \in[0,1]$ be irrational. If $r \in Q \cap[0,1]$ and $b<r$ then by additivity we obtain

$$
a \circ b \leq a \circ r=a r
$$

Similarly, if $r \in Q \cap[0,1]$ and $b>r$, then

$$
a \circ b \geq a \circ r=a r
$$

Since $Q \cap[0,1]$ is dense in $[0,1]$, we obtain $a \circ b=a b$. 
The next theorem formalizes the obvious observation that if two effect algebras are isomorphic and one admits an operation satisfying some special conditions, then so does the other.

Theorem 4.2. Let $E, F$ be effect algebras and let $\phi: E \rightarrow F$ be an effect algebra isomorphism [6, 7]. If $\circ$ is a sequential product on $E$, then $a * b=$ $\phi\left[\phi^{-1}(a) \circ \phi^{-1}(b)\right]$ is a sequential product on $F$. Moreover, $(E, \circ)$ and $(F, *)$ are SEA isomorphic [13].

Proof. The proof is a straightforward verification.

Corollary 4.3. If $E$ and $F$ are isomorphic effect algebras and $E$ admits a unique sequential product $\circ$, then $F$ admits a unique sequential product.

Proof. By Theorem 4.2, $F$ admits a sequential product $*$. Let $\phi: E \rightarrow F$ be an effect algebra isomorphism and define $\bullet: E \times E \rightarrow E$ by $a \bullet b=\phi^{-1}[\phi(a) * \phi(b)]$. By Theorem 1.2, $\bullet$ is a sequential product on $E$ so that $a \bullet b=a \circ b$. Hence,

$$
\phi(a \circ b)=\phi(a \bullet b)=\phi(a) * \phi(b)
$$

thus, for every $c, d \in F$ we have

$$
c * d=\phi\left[\phi^{-1}(c)\right] * \phi\left[\phi^{-1}(d)\right]=\phi\left[\phi^{-1}(c) \circ \phi^{-1}(d)\right]
$$

It follows that $*$ is unique.

Corollary 4.4. If an effect algebra $E$ admits a unique sequential product $\circ$, then any effect algebra automorphism $\phi: E \rightarrow E$ is a $S E A$ automorphism.

Proof. By Theorem 4.2, $a * b=\phi^{-1}[\phi(a) \circ \phi(b)]$ is a sequential product on $E$. Hence, $a * b=a \circ b$ and the result follows.

We say that $a, b \in E$ coexist if there exist $c, d, e \in E$ such that $c \oplus d \oplus e$ is defined and $a=c \oplus d, b=c \oplus e$.

Lemma 4.5. Let $\circ$ and $*$ be sequential products on an effect algebra $E$ and let $b \in E_{S}$. If $a \circ b=b \circ a$, then $a * b=b * a$.

Proof. It is shown in 13 that $a \circ b=b \circ a$ if and only if $a$ and $b$ coexist. But coexistence is independent of the sequential product.

Theorem 4.6. Let $\left(E_{i}, 0_{i}, 1_{i}, \oplus_{i}, \circ_{i}\right)$ be $S E A$ 's, $i \in I$. Then $E=\Pi E_{i}$ admits a unique sequential product if and only if each $E_{i}, i \in I$, admits a unique sequential product.

Proof. If $E_{j}$ admits two sequential products for some $j \in I$, then clearly $\Pi E_{i}$ admits at least two sequential products. Conversely, suppose $E_{i}, i \in I$, admits a unique sequential product $\circ_{i}$ and let $*$ be a sequential product on $\Pi E_{i}$. For $j \in I$, let $f_{j} \in \Pi E_{i}$ be define by

$$
f_{j}(i)= \begin{cases}1_{j} & \text { if } i=j \\ {[.25 p c] 0_{i}} & \text { if } i \neq j\end{cases}
$$


Clearly, $f_{j} \in E_{S}$. Let $\circ$ be the sequential product on $E$ given by $(f \circ g)(i)=$ $f(i) \circ_{i} g(i), i \in I$, for any $f, g \in E$. Since $f \circ f_{j}=f_{j} \circ f$, by Lemma 4.5, $f * f_{j}=f_{j} * f$ for any $f \in E$. It follows from Theorem 3.4 [13] that $f * f_{j}=f \wedge f_{j}$. Hence,

$$
f * f_{j}(i)= \begin{cases}0_{i} & \text { if } i \neq j \\ {[.25 p c] f(j)} & \text { if } i=j\end{cases}
$$

For any $f, g \in E$ we have

$$
\begin{aligned}
(f * g) * f_{j} & =f_{j} *(f * g)=\left(f_{j} * f\right) * g=\left[\left(f * f_{j}\right) * f_{j}\right] * g \\
& =\left(f * f_{j}\right) *\left(f_{j} * g\right)
\end{aligned}
$$

Now $\left[0, f_{j}\right] \subseteq E$ is an effect algebra with greatest element $f_{j}$ and $\phi: E_{j} \rightarrow\left[0, f_{j}\right]$ given by

$$
[\phi(a)](i)= \begin{cases}0_{i} & \text { if } i \neq j \\ {[.25 p c] a} & \text { if } i=j\end{cases}
$$

is an effect algebra isomorphism. Since $E_{j}$ admits a unique sequential product, by Corollary $4.3,\left[0, f_{j}\right]$ admits a unique sequential product. Hence,

$$
\begin{aligned}
(f * g)(j) & =\left[(f * g) * f_{j}\right](j)=\left[\left(f * f_{j}\right) *\left(g * f_{j}\right)\right](j) \\
& =\left[\left(f * f_{j}\right) \circ\left(g * f_{j}\right)\right](j)=\left(f * f_{j}\right)(j) \circ_{j}\left(g * f_{j}\right)(j) \\
& =f(j) \circ_{j} g(j)=(f \circ g)(j)
\end{aligned}
$$

Hence, $\circ$ is the unique sequential product on $E$.

Corollary 4.7. A full fuzzy set system $E=[0,1]^{X}$ admits a unique sequential product.

Proof. This follows from Theorem 4.6 because, by Theorem 4.1, $[0,1]$ admits a unique sequential product

The next result characterizes the only known sequential product on $\mathcal{E}(H)$. For $x \in H$ with $\|x\|=1, P_{x}$ denoted the one-dimensional projection onto the span of $x$.

Theorem 4.8. Let $\circ: \mathcal{E}(H) \times \mathcal{E}(H) \rightarrow \mathcal{E}(H)$ be a binary operation. Then $A \circ B=A^{1 / 2} B A^{1 / 2}$ for every $A, B \in \mathcal{E}(H)$ if and only if the following conditions are satisfied: (1) $B \mapsto A \circ B$ is $\sigma$-additive in the strong operator topology for every $A \in \mathcal{E}(H) ;(2)(\lambda A) \circ B=\lambda(A \circ B)$ for every $\lambda \in[0,1]$; (3) there exists a Borel function $f:[0,1] \rightarrow[0,1]$ such that $f(1)=1$ and $\left\langle A \circ P_{x} y, y\right\rangle=$ $|\langle f(A) x, y\rangle|^{2}$ for every $A \in \mathcal{E}(H), x, y \in H$ with $\|x\|=\|y\|=1$.

Proof. We have already observed that $A \circ B=A^{1 / 2} B A^{1 / 2}$ is a sequential operation on $\mathcal{E}(H)$; that these properties hold for this operation is straightforward, 
with $f(\lambda)=\lambda^{1 / 2}$ in (3). To prove the converse, assume the conditions and observe that, by (2) and (3), for every $\lambda \in[0,1]$ we have

$$
\begin{aligned}
|\langle f(\lambda A) x, y\rangle|^{2} & =\left\langle(\lambda A) \circ P_{x} y, y\right\rangle=\lambda\left\langle A \circ P_{x} y, y\right\rangle=\lambda|\langle f(A) x, y\rangle|^{2} \\
& =\left|\left\langle\lambda^{1 / 2} f(A) x, y\right\rangle\right|^{2}
\end{aligned}
$$

Letting $y=x$ gives $\langle f(\lambda A) x, x\rangle=\left\langle\lambda^{1 / 2} f(A) x, x\right\rangle$ for every $x \in H$ with $\|x\|=1$. Hence, $f(\lambda A)=\lambda^{1 / 2} f(A)$. Letting $A=I$ gives

$$
f(\lambda) I=f(\lambda I)=\lambda^{1 / 2} f(I)=\lambda^{1 / 2} I
$$

so that $f(\lambda)=\lambda^{1 / 2}$. Thus,

$$
\left\langle A \circ P_{x} y, y\right\rangle=\left|\left\langle A^{1 / 2} x, y\right\rangle\right|^{2}=\left\langle A^{1 / 2} P_{x} A^{1 / 2} y, y\right\rangle
$$

for every $y \in H$ with $\|y\|=1$. It follows that $A \circ P_{x}=A^{1 / 2} P_{x} A^{1 / 2}$. By (1) we have $A \circ P=A^{1 / 2} P A^{1 / 2}$ for every $P \in \mathcal{P}(H)$. As in the proof of Theorem 4.1 we have $A \circ(\lambda B)=\lambda A \circ B$ for every $\lambda \in[0,1]$. Hence, by (1) we conclude that $A \circ B=A^{1 / 2} B A^{1 / 2}$ for every $B \in \mathcal{E}(H)$ with finite spectrum. Since any $B \in \mathcal{E}(H)$ is the strong limit of an increasing sequence of $B_{i} \in \mathcal{E}(H)$ with finite spectra, it follows from (1) that $A \circ B=A^{1 / 2} B A^{1 / 2}$ for every $B \in \mathcal{E}(H)$.

Since the sequential product $A \circ B=A^{1 / 2} B A^{1 / 2}$ is the only known sequential product in $\mathcal{E}(H)$, we shall refer to it as the standard sequential product on $\mathcal{E}(H)$. When we refer to $\mathcal{E}(H)$ as a SEA, without reference to a specific sequential product, we mean with respect to the standard sequential product.

\section{Sequentially Ordered SEA's}

An effect algebra $E$ is sharply dominating if for every $a \in E$ there exists a least element $\widehat{a} \in E_{S}$ such that $a \leq \widehat{a}[9]$. A sharply dominating SEA is sequentially ordered if (1) $a \leq b$ implies that there exists a $c \in E$ such that $a=b \circ c$ and (2) if $c \circ a \leq c \circ b$ then $\widehat{c} \circ a \leq \widehat{c} \circ b$. Notice that the converses of (1) and (2) hold for any SEA. Condition (1) states that the order is completely determined by $\circ(a \leq b$ if and only if $a=b \circ c$ for some $c)$. This is similar to order being completely determined by $\oplus(a \leq b$ if and only if $a \oplus c=b$ for some $c)$. It is easy to check that Boolean algebras and $[0,1] \subseteq \mathbb{R}$ are sequentially ordered.

Example 3 (continued). Let $E=[0,1]^{X}$ be a full fuzzy set system. For $f \in E$ let

$$
\operatorname{supp}(f)=\{x \in X: f(x) \neq 0\}
$$

and define $\widehat{f}$ to be the characteristic function on $\operatorname{supp}(f)$. Then $\widehat{f}$ is the least sharp element that dominates $f$ so $E$ is sharply dominating. If $f \leq g$ then 
$f=g h$ where

$$
h(x)= \begin{cases}f(x) / g(x) & \text { if } g(x) \neq 0 \\ {[.25 p c] 0} & \text { if } g(x)=0\end{cases}
$$

Hence, $E$ satisfies Condition (1). If $h f \leq h g$, then $f(x) \leq g(x)$ for all $x \in \operatorname{supp}(h)$ so $\widehat{h} f \leq \widehat{h} g$. Hence, $E$ satisfies Condition (2) so $E$ is sequentially ordered.

Now let $F$ be the fuzzy set system of all polynomial functions $f:[0,1] \rightarrow[0,1]$. Then $F_{S}=\{0,1\}$ and $F$ is sharply dominating. But $F$ does not satisfy (1) so $F$ is not sequentially ordered. Indeed, the functions $f(x)=\frac{1}{2} x$ and $g(x)=\frac{1}{2}+\frac{1}{2} x$ are in $F$ and $f \leq g$. Suppose there exists an $h \in F$ such that $f=g h$. Then $h(x)=x /(x+1)$ on $[0,1]$ but $h \notin F$ which is a contradiction. However, $F$ does satisfy (2). Indeed, if $h f \leq h g$ then $f(x) \leq g(x)$ for all $x \in \operatorname{supp}(h)$. But if $h \neq 0$, then $h(x)=0$ for only a finite number of points $x_{i} \in[0,1], i=1, \ldots, n$. If $f\left(x_{i}\right)>g\left(x_{i}\right)$ for some $i$, then by continuity $f(x)>g(x)$ in a neighborhood of $x_{i}$ which is a contradiction. Hence,

$$
\widehat{h} f=f \leq g=\widehat{h} g
$$

Example 4 (continued). It is well known that $\mathcal{E}(H)$ is sharply dominating [9. We shall show in the next theorem that $\mathcal{E}(H)$ is sequentially ordered.

Example 6 (continued). The SEA $\omega+\omega^{*}$ is sharply dominating with $\left(\omega+\omega^{*}\right)_{S}=\{0,1\}$. However, $\omega+\omega^{*}$ is not sequentially ordered. Indeed, $a \leq 2 a$ but there is no $c \in \omega+\omega^{*}$ such that $a=(2 a) \circ c$. Also, (2) does not hold because $a \circ 2 a=0=a \circ a$ but

$$
\widehat{a} \circ 2 a=2 a \not \leq a=\widehat{a} \circ a
$$

Example 7 (continued). It is easy to check that $E=H S(\mathcal{E}(H),[0,1])$ is sharply dominating and satisfies (1). However, $E$ does not satisfy (2). Indeed, if $a \in(0,1)$ then $a \circ A \leq a \circ B$ if and only if $\operatorname{tr}(W A) \leq \operatorname{tr}(W B)$ but this does not imply that

$$
\widehat{a} \circ A=A \leq B=\widehat{a} \circ B
$$

This observation together with the second part of Example 3 shows that Conditions (1) and (2) are logically independent.

Theorem 5.1. The $S E A \mathcal{E}(H)$ is sequentially ordered.

Proof. For $A \in \mathcal{E}(H)$ let $P_{A}$ be the projection onto the closure of the range $\bar{R}(A)$ of $A$. Then $P_{S} \in \mathcal{P}(H)=\mathcal{E}(H)_{S}$ and it is easy to see that $P_{A}$ is the least sharp element satisfying $A \leq P_{A}$. Hence, $P_{A}=\widehat{A}$ and $\mathcal{E}(H)$ is sharply dominating. We now show that if $C \in \mathcal{E}(H)$, then $\bar{R}(C)=\bar{R}\left(C^{1 / 2}\right)$. If $C x=0$, then

$$
\left\langle C^{1 / 2} x, C^{1 / 2} x\right\rangle=\langle C x, x\rangle=0
$$


so that $C^{1 / 2} x=0$. Hence, $\operatorname{Ker}(C) \subseteq \operatorname{Ker}\left(C^{1 / 2}\right)$. Conversely, if $C^{1 / 2} x=0$ then $C x=0$ so $\operatorname{Ker}\left(C^{1 / 2}\right) \subseteq \operatorname{Ker}(C)$. Hence, $\operatorname{Ker}(C)=\operatorname{Ker}\left(C^{1 / 2}\right)$ and we have

$$
\bar{R}(C)=\operatorname{Ker}(C)^{\perp}=\operatorname{Ker}\left(C^{1 / 2}\right)^{\perp}=\bar{R}\left(C^{1 / 2}\right)
$$

Now suppose that $C \circ A \leq C \circ B$. Then for any $x \in H$ we have

$$
\left\langle A C^{1 / 2} x, C^{1 / 2} x\right\rangle \subseteq\left\langle B C^{1 / 2} x, C^{1 / 2} x\right\rangle
$$

Hence, $\langle A y, y\rangle \leq\langle B y, y\rangle$ for any $y \in \bar{R}\left(C^{1 / 2}\right)=\bar{R}(C)$. We conclude that

$$
\left\langle A P_{C} x, P_{C} x\right\rangle \leq\left\langle B P_{C} x, P_{C} x\right\rangle
$$

for any $x \in H$. Hence,

$$
\widehat{C} \circ A=P_{C} A P_{C} \leq P_{C} B P_{C}=\widehat{C} \circ B
$$

so Condition (2) holds. To verify Condition (1) suppose that $A \leq B$. Then $A^{1 / 2} A^{1 / 2} \leq B^{1 / 2} B^{1 / 2}$ and it follows from $[5$ that there exists a bounded linear operator $T$ on $H$ such that $\|T\| \leq 1$ and $A^{1 / 2}=B^{1 / 2} T$. Letting $C=T T^{*}$, we see that $C \geq 0$. Moreover, for any $x \in H$ we have

$$
\langle C x, x\rangle=\left\langle T^{*} x, T^{*} x\right\rangle=\left\|T^{*} x\right\|^{2} \leq\left\|T^{*}\right\|^{2}\|x\|^{2} \leq\|x\|^{2}=\langle x, x\rangle
$$

so that $C \in \mathcal{E}(H)$. Hence,

$$
A=A^{1 / 2}\left(A^{1 / 2}\right)^{*}=B^{1 / 2} T T^{*} B^{1 / 2}=B^{1 / 2} C B^{1 / 2}=B \circ C
$$

Theorem 5.2. Let $E$ be a sequentially ordered $S E A$. For $a, b \in E$ with $a \leq b$ there exists a unique $c \in E$ such that $c \leq \widehat{b}$ and $a=b \circ c$.

Proof. By Condition (1) there is a $d \in E$ such that $a=b \circ d$. Letting $c=\widehat{b} \circ d$ we have $c \leq \widehat{b}$ and

$$
a=b \circ d=(b \circ \widehat{b}) \circ d=b \circ(\widehat{b} \circ d)=b \circ c
$$

For uniqueness, suppose that $c_{1} \leq \widehat{b}$ and $a=b \circ c_{1}$. Then $b \circ c_{1}=b \circ c$ and applying Condition (2) we have

$$
c_{1}=\widehat{b} \circ c_{1}=\widehat{b} \circ c=c
$$

We denote the unique element $c$ in Theorem 5.2 by $c=a / b$ and call $c$ the sequential quotient of $a$ over $b$. Thus, / is a partial binary operation on $E$ with domain $\{(a, b): a \leq b\}$.

Corollary 5.3. Let $E$ be a sequentially ordered SEA. (i) For every $a, b, c \in E$ there exists a unique $d \in E$ such that $d \leq(a \circ b)^{\wedge}$ and $a \circ(b \circ c)=(a \circ b) \circ d$. (ii) $a \leq b$ if and only if there exists a unique $d \in E$ such that $d \geq(\widehat{b})^{\prime}$ and $a \oplus b \circ d=b$. 
Proof. (i) Since $b \circ c \leq b$ we have $a \circ(b \circ c) \leq a \circ b$. By Theorem 5.2 there exists a unique $d \in E$ such that $d \leq(a \circ b)^{\wedge}$ and $a \circ(b \circ c)=(a \circ b) \circ d$. (ii) If $a \leq b$, then by Theorem 5.2 there exists a unique $c \in E$ (namely, $c=a / b$ ) such that $c \leq \widehat{b}$ and $a=b \circ c$. Hence, $c^{\prime} \geq(\widehat{b})^{\prime}$ and

$$
b=b \circ c \oplus b \circ c^{\prime}=a \oplus b \circ c^{\prime}
$$

with the uniqueness of $c^{\prime}$ following from the uniqueness of $c$. The converse is clear.

The proof of the next lemma is straightforward.

Lemma 5.4. Let $E$ be a sequentially ordered $S E A$ and let $a \in E$. (i) $a / a=\widehat{a}$. (ii) $a \in E_{S}$ if and only if $a / a=a$. (iii) Let $b \in E_{S}$. If $a \leq b$ then $a / b=a$ and if $b \leq a$ then $b / a=b$. In particular, $a / 1=a$ and $0 / a=0$ for every $a \in E$. (iv) If $n \geq 1$, then $a^{n+m} / a^{m}=a^{n}$.

If $a \leq b$ then the unique $c$ such that $a \oplus c=b$ is denoted by $b \ominus a$.

Theorem 5.5. Let $E$ be a sequentially ordered $S E A$ with $a, b, c \in E$. (i) If $a \leq b$ then $(b \ominus a) / b=\widehat{b} \circ(a / b)^{\prime}$. (ii) $(a \circ b) / a=\widehat{a} \circ b$. (iii) If $a \leq b \leq c$ then $a / c \leq b / c$. (iv) Let $a, b \leq c$. Then $a \oplus b \leq c$ iff $(a / c) \perp(b / c)$, and in this case $(a \oplus b) / c=a / c \oplus b / c$. (v) $a /(a \oplus b)=(a \oplus b)^{\wedge} \circ[b /(a \oplus b)]^{\prime}$. (vi) If $a \leq b$ and $b \mid(a / b)$ then $b \mid a$.

Proof. (i) For $a \leq b$ we have

$$
b \circ\left[\widehat{b} \circ(a / b)^{\prime}\right]=b \circ(a / b)^{\prime}=b \circ(1 \ominus a / b)=b \ominus b \circ(a / b)=b \ominus a
$$

and $\widehat{b} \circ(a / b)^{\prime} \leq \widehat{b}$. (ii) Since $a \circ b=a \circ(\widehat{a} \circ b)$ and $\widehat{a} \circ b \leq \widehat{a}$ we have $(a \circ b) / a=\widehat{a} \circ b$. (iii) Since $a=c \circ(a / c)$ and $b=c \circ(b / c)$ we have $c \circ(a / c) \leq c \circ(b / c)$. Applying Condition (2) gives

$$
a / c=\widehat{c} \circ(a / c) \leq \widehat{c} \circ(b / c)=b / c
$$

(iv) If $a \oplus b \leq c$, then $a, b \leq c$ and both $a / c$ and $b / c$ are defined. Since $a \leq c \ominus b$, by (i) and (iii) we have

$$
a / c \leq(c \ominus b) / c=\widehat{c} \circ(b / c)^{\prime}=\widehat{c} \ominus(b / c)
$$

Hence, $a / c \oplus b / c$ is defined. Now

$$
a \oplus b=c \circ(a / c) \oplus c \circ(b / c)=c \circ(a / c \oplus b / c)
$$

Since, by Lemma 4.2 of [13], $a / c \oplus b / c \leq \widehat{c}$ we have $(a \oplus b) / c=a / c \oplus b / c$. If $(a / c) \perp(b / c)$ then, since

$$
c \circ(a / c \oplus b / c)=c \circ(a / c) \oplus c \circ(b / c)=a \oplus b
$$

we have $a \oplus b \leq c$. The result now follows from the above.

(v) This follows from (i).

(vi) This follows because $b \circ a=b \circ[b \circ(a / b)]=[b \circ(a / b)] \circ b=a \circ b$ 
The next result shows that the condition in Theorem 5.5(ii) characterizes the sequential quotient.

Lemma 5.6. Let $E$ be a sequentially ordered $S E A$. If // is a partial binary operation on $E$ with domain $\{(a, b): a \leq b\}$ and $(a \circ b) / / a=\widehat{a} \circ b$ for every $a, b \in E$, then // and / coincide.

Proof. If $a \leq b$ then $a=b \circ c$ where $c \leq \widehat{b}$. Hence

$$
a / / b=b \circ c / / b=\widehat{b} \circ c=c=a / b
$$

Let $E$ be an effect algebra and let $b \in E$ with $b \neq 0$. Then we have seen in Section 2 that $\left([0, b], 0, b, \oplus_{b}\right)$ is an effect algebra. If $E$ is also a SEA, does $[0, b]$ admit a sequential product? If $b \in E_{S}$ the answer is yes. Just restrict $\circ$ to $[0, b]$. In this case, $b \circ a=a$ for all $a \in[0, b]$ and the other axioms are easily verified so that $\left([0, b], 0, b, \oplus_{b}, \circ\right)$ is a SEA. In general, the answer is no. For example, in $\omega+\omega^{*}$ the interval $[0,2 a]=\{0, a, 2 a\}$ is isomorphic to $C_{3}$ so $[0,2 a]$ does not admit a sequential product. We now show that the answer is positive if $E$ is sequentially ordered.

Let $E$ be a sequentially ordered SEA and let $b \in E$ with $b \neq 0$. Define $\phi_{b}:[0, b] \rightarrow[0, \widehat{b}]$ by $\phi_{b}(a)=a / b$.

Lemma 5.7. The map $\phi_{b}:[0, b] \rightarrow[0, \widehat{b}]$ is an effect algebra isomorphism.

Proof. By Theorem 5.5(iv), if $a, c \in[0, b]$ and $a \oplus c \leq b$ then

$$
\phi_{b}(a \oplus c)=(a \oplus c) / b=a / b \oplus c / b=\phi_{b}(a) \oplus \phi_{b}(c)
$$

Hence, $\phi_{b}$ is additive. Also, $\phi_{b}(b)=\widehat{b}$ so $\phi_{b}$ is a morphism [6, 7]. If $\phi_{b}(a) \perp \phi_{b}(c)$ then by Theorem 5.5(v), $a \oplus c \leq b$ so $a \perp c$. Thus, $\phi_{b}$ is a monomorphism [6, 7]. If $c \in[0, \widehat{b}]$, letting $a=b \circ c$ we have $a \in[0, b]$ and $\phi_{b}(a)=a / b=c$. Hence, $\phi_{b}$ is surjective so $\phi_{b}$ is an effect algebra isomorphism.

Theorem 5.8. Let $E$ be a sequentially ordered $S E A$ and let $b \in E$ with $b \neq 0$. (i) There exists a unique sequential product $o_{b}$ on $[0, b]$ such that

$$
\left(a \circ_{b} c\right) / b=(a / b) \circ(c / b)
$$

(ii) Employing this sequential product on $[0, b], \phi_{b}:[0, b] \rightarrow[0, \widehat{b}]$ becomes a SEA isomorphism.

Proof. (i) Uniqueness follows from $a \circ_{b} c=b \circ[(a / b) \circ(c / b)]$. By Lemma 5.7, $\phi_{b}:[0, b] \rightarrow[0, \widehat{b}]$ is an effect algebra isomorphism. Letting $\psi=\phi_{b}^{-1}$ we conclude, using Theorem 5.5 part (ii), that $\psi:[0, \widehat{b}] \rightarrow[0, b]$ is an effect algebra isomorphism given by $\psi(a)=b \circ a$. Applying Theorem 4.2, for $a, c \in[0, b]$ we have

$$
a * c=\psi\left[\psi^{-1}(a) \circ \psi^{-1}(b)\right]=b \circ\left[\phi_{b}(a) \circ \phi_{b}(c)\right]=b \circ[(a / b) \circ(c / b)]
$$

is a sequential product on $[a, b]$. (ii) This follows from Theorem 4.2 . 


\section{References}

[1] M. K. Bennett and D. J. Foulis, Interval and scale effect algebras, Adv. Appl. Math. 91, (1997), 200-215.

[2] P. Busch, P. J. Lahti and P. Middlestaedt, The Quantum Theory of Measurements, Springer-Verlag, Berlin, 1991.

[3] P. Busch and J. Singh, Lüders theorem for unsharp effects, Phys. Lett. A, 249 (1998), 10-24.

[4] E. B. Davies, Quantum Theory of Open Systems, Academic Press, New York, 1976.

[5] R. G. Douglas, On majorization, factorization, and range inclusion of operators on Hilbert space, Proc. Amer. Math. Soc., 17 (1966), 413-415.

[6] A. Dvurečenskij and S. Pulmannová, New Trends in Quantum Structures, Kluwer, Dordrecht, 2000.

[7] D. J. Foulis and M. K. Bennett, Effect algebras and unsharp quantum logics, Found. Phys. 24, (1994), 1325-1346.

[8] R. Giuntini and H. Greuling, Toward a formal language for unsharp properties, Found. Phys. 19 (1989), 931-945.

[9] S. Gudder, Sharply dominating effect algebras, Tatra Mt. Math. Publ. 15 (1998), 23-30.

[10] S. Gudder, A histories approach to quantum mechanics, J. Math. Phys. 39 (1998), 5772-5788.

[11] S. Gudder and G. Nagy, Sequentially independent effects, Proc. Amer. Math. Soc. 130 (2001), 1125-1130.

[12] S. Gudder and G. Nagy, Sequential quantum measurements, J. Math. Phys. 42 (2001), 5212-5222.

[13] S. Gudder and R. Greechie, Sequential products on effect algebras, Rep. Math. Phys. 49 (2002), 87-111.

[14] F. Kôpka and F. Chovanec, D-Posets Math. Slovaca 44 (1994), 21-34.

[15] K. Kraus, States, Effects, and Operations, Springer-Verlag, Berlin, 1983.

[16] G. Ludwig, Foundations of Quantum Mechanics, Springer-Verlag, Berlin, 1983. 